\title{
Synergistic protective effects of escin and low-dose glucocorticoids on blood-retinal barrier breakdown in a rat model of retinal ischemia
}

\author{
FENGLAN ZHANG ${ }^{1,2}$, YUANBIN LI $^{2}$, LEIMING ZHANG ${ }^{3}$ and GUOYING MU ${ }^{1}$ \\ ${ }^{1}$ Department of Ophthalmology, Provincial Hospital Affiliated to Shandong University, Jinan, Shandong 250021; \\ ${ }^{2}$ Department of Ophthalmology, Yantai Yuhuangding Hospital, Yantai, Shandong 264001; \\ ${ }^{3}$ Department of Pharmacology, School of Pharmacy, Yantai University, Yantai, Shandong 264005, P.R. China
}

Received January 10, 2013; Accepted March 13, 2013

DOI: $10.3892 / \mathrm{mmr} .2013 .1374$

\begin{abstract}
Escin, a natural mixture of triterpenoid saponins isolated from the seed of the horse chestnut (Aesculus hippocastanum), has been demonstrated to possess glucocorticoid (GC)-like anti-edematous and anti-inflammatory effects. The aim of the present study was to investigate whether escin exhibits synergistic protective effects on blood-retinal barrier (BRB) breakdown when combined with GCs in a rat model of retinal ischemia. Low concentrations of escin and triamcinolone acetonide (TA) alone did not affect BRB permeability. However, when administered together, low-dose escin and TA significantly reduced BRB permeability following ischemia. Furthermore, low-dose escin and TA alone did not affect the expression of occludin in the ischemic retina; however, when administered together, they significantly increased occludin expression in the ganglion cell layer of the ischemic retina. This indicates that escin and GCs have synergistic protective effects on BRB breakdown and the molecular mechanisms may be correlated with the upregulation of occludin. Therefore, the administration of escin may allow a reduction in the dose of GCs for the treatment of macular edema. The combination of escin with GCs is potentially a beneficial treatment method for BRB breakdown and warrants further investigation.
\end{abstract}

\section{Introduction}

Numerous diseases cause breakdown of the blood-retinal barrier (BRB) and macular edema. Hypoxia, altered blood flow and retinal ischemia are closely correlated with the development and progression of macular edema $(1,2)$.

Correspondence to: Professor Guoying $\mathrm{Mu}$, Department of Ophthalmology, Provincial Hospital Affiliated to Shandong University, 324 Jingwu Road, Jinan, Shandong 250021, P.R. China E-mail: mgyeyes@yahoo.com.cn

Key words: escin, blood-retinal barrier, glucocorticoids, occludin
Currently, glucocorticoids (GCs) are the most commonly used medicine for ocular applications. Recent studies have demonstrated the efficacy and complications associated with intravitreal GC injection for diabetic macular edema $(3,4)$. GCs may act by suppressing inflammation and directly affecting endothelial cells, through regulation of the phosphorylation, organization and content of tight junction proteins. GCs have also been demonstrated to reduce the breakdown of the BRB (5). It has been identified that GCs upregulate the tight junction transmembrane proteins, occludin and claudin 5 , in primary retinal endothelial cells through transactivation of the promoters for these genes (6).

Although GCs have been demonstrated to ameliorate macular edema, the side-effects accompanying treatment with GCs cause their frequent use to be problematic. One of the major side-effects of intravitreal injection of triamcinolone acetonide (TA), a synthetic corticosteroid, is steroid-induced increases in intraocular pressure (IOP). One study demonstrated that a rise in IOP to values $>21 \mathrm{mmHg}$ may be expected to occur in $\sim 50 \%$ of treated eyes (7). The frequency of cataract surgery following intravitreal injection of high-dose TA in elderly patients is increasing (8).

Escin is a natural mixture of triterpene saponins, mainly consisting of A-, B-, C- and D-escin (Fig. 1). Accumulating experimental evidence suggests that escin exerts anti-inflammatory and anti-edematous effects. Escin inhibits acetic acid-induced increases in capillary permeability and adhesion formation in animal models. It also attenuates cognitive deficits and hippocampal injury following transient global cerebral ischemia in mice, by regulating certain inflammatory genes $(9,10)$. Furthermore, escin has been demonstrated to be a safe and potent anti-inflammatory drug with long-term, effective anti-inflammatory action without immunosuppression $(11,12)$. Escin also exerts synergistic anti-inflammatory effects with low doses of GCs in vivo and in vitro (13). In China, escin is widely used (clinically) for the treatment of retinal vein occlusion and central serous chorioretinopathy (14-16).

In the present study, we investigated whether the combination of escin and GCs was able to produce synergistic protective effects on BRB breakdown in a rat model of acute retinal ischemia. 


\section{Materials and methods}

Subjects and drugs. Male Sprague-Dawley rats (weight, $180 \pm 20 \mathrm{~g}$ ) were obtained from the Experimental Animal Center of Luye Pharma Group (Yantai, China). Rats were kept in an air-conditioned room (temperature, $23 \pm 2^{\circ} \mathrm{C}$ ) with a 12-h light-dark cycle, with free access to food and water. Animal experimental procedures were conducted in strict accordance with the National Institutes of Health regulations on the use and care of animals for scientific purposes (NIH Publication No. 0-23, revised 1996). Surgical procedures and the sacrifice of animals (at the end of the observation period) were performed under general anesthesia induced by an intraperitoneal (i.p.) injection of chloral hydrate $(350 \mathrm{mg} / \mathrm{kg})$.

Sodium salt of escin (sodium escinate), consisting of A-, B-, C- and D-escin, was obtained from Shandong Luye PharmaceuticalCo.,Ltd.(batch no. 1206043; Yantai, China) and TA injections were provided by Kunming Jida Pharmaceutical Co., Ltd. (batch no. 120105; Kunming, China). Evans blue dye (Sinopharm Chemical Reagent Co., Ltd, Shanghai, China) was prepared by dissolving the dye in normal saline $(45 \mathrm{mg} / \mathrm{ml})$.

Retinal ischemia-reperfusion study protocol. Rats were anesthetized intraperitoneally with chloral hydrate $(350 \mathrm{mg} / \mathrm{kg})$ and chlorpromazine ( $3 \mathrm{mg} / \mathrm{kg}$ ). Five minutes following administration of the anesthetics, the anterior chamber of the left eye was cannulated with a 30-gauge needle connected by silicone elastomer tubing to a reservoir of balanced oxyglutathione solution. The IOP was raised to $120 \mathrm{mmHg}$ by increasing the height of reservoir. The eye was examined with an ophthalmoscope to confirm that the retinal vessels had collapsed and the retina appeared gray, indicating that the retina was ischemic. After $50 \mathrm{~min}$ of retinal ischemia, the IOP had returned to normal and ophthalmoscopy was used to confirm that the circulation had also returned to normal. The body temperature of the rats was maintained at $37^{\circ} \mathrm{C}$ with a heating pad during ischemia, until the rats had recovered from anesthesia.

Following surgery, the rats were divided randomly into four groups: the ischemia-reperfusion (IR); TA (2 and $5 \mu \mathrm{l})$ treatment; escin $(0.9$ and $1.8 \mathrm{mg} / \mathrm{kg})$ treatment; and combined escin $(0.9 \mathrm{mg} / \mathrm{kg})$ and TA $(2 \mu \mathrm{l})$ treatment groups. Following the induction of general anesthesia, pupils were dilated with $0.5 \%$ tropicamide. The rats were treated with escin through the caudal vein and with TA through the vitreous body. A group of rats underwent surgery without increased IOP, in order to serve as a sham group. Twenty-four hours after surgery, the BRB permeability was assayed.

Evaluation of BRB permeability. Following the induction of general anesthesia with chloral hydrate $(350 \mathrm{mg} / \mathrm{kg})$, Evans blue was injected into the blood through the caudal vein at a dosage of $45 \mathrm{mg} / \mathrm{kg}$. When the dye had circulated for $120 \mathrm{~min}$, the chest cavity was opened and the rats were perfused via the left ventricle with citrate buffer $(0.05 \mathrm{M}, \mathrm{pH} 3.5)$ for $2 \mathrm{~min}$ at $37^{\circ} \mathrm{C}$. Immediately after perfusion, both eyes were enucleated and bisected at the equator. The retinas were then carefully dissected away. Following measurement of the retinal weight, Evans blue was extracted by incubating each retina in $180 \mu \mathrm{l}$ formamide for $18 \mathrm{~h}$ at $70^{\circ} \mathrm{C}$. The extract was ultracentrifuged for $60 \mathrm{~min}$ at $12,000 \mathrm{x} \mathrm{g}$ and $25^{\circ} \mathrm{C}$. In total, $60 \mu 1$ supernatant
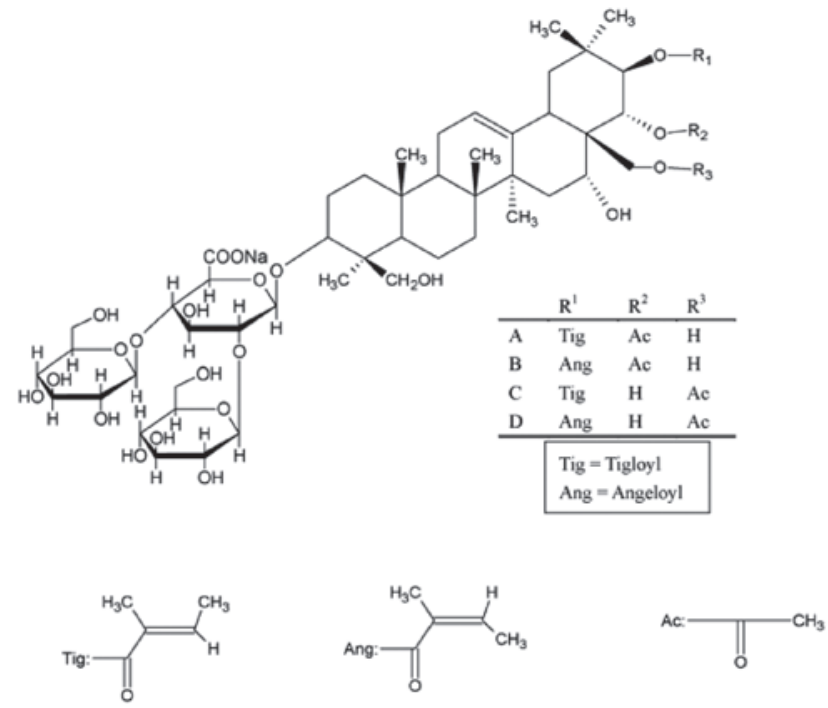

Figure 1. Structure of escin.

was used for spectrophotometry. The background-subtracted absorbance was determined by measuring the absorbance of each sample at $620 \mathrm{~nm}$ (the absorbance maximum for Evans blue in formamide) and at $740 \mathrm{~nm}$ (the absorbance minimum). BRB breakdown was calculated as follows: Evans blue weight $(\mathrm{ng}) \mathrm{x}$ retinal weight $(\mathrm{mg})^{-1}$.

Morphological analysis. Retinal thickness was evaluated in hematoxylin and eosin-stained sections using the Metamorph/BX51 Microscopic image analysis software (Molecular Devices, USA). The eyes were cut vertically through the center of the cornea and optic nerve, and the two halves were embedded face down. Care was taken to maintain a consistent cutting angle for all the eyes measured for retinal thickness. Images of retinal sections were captured and retinal thickness was measured under the same conditions.

Immunohistochemistry. Twenty-four hours after renal ischemia, rats were deeply anesthetized and transcardially perfused with saline solution, followed by $4 \%$ paraformaldehyde in $0.1 \mathrm{M}$ phosphate-buffered saline (PBS) for $2 \mathrm{~min}$. The eyes were enucleated and post-fixed in $4 \%$ paraformaldehyde for $24 \mathrm{~h}$. Coronal sections $(10 \mu \mathrm{m})$ were obtained using a Leica CM1950S cryostat (Leica Microsystems, Wetzlar, Germany). Sections were blocked with $3 \%$ normal goat serum (diluted in PBS containing $0.3 \%$ Triton X-100) for $1 \mathrm{~h}$ and incubated with primary antibodies (anti-occludin; 1:200; Abcam, Cambridge, MA, USA) overnight at $4^{\circ} \mathrm{C}$. Following rinsing with $\mathrm{PBS}$, sections were incubated with horseradish peroxidase-conjugated goat anti-rabbit IgG secondary antibody (1:200; Beyotime Institute of Biotechnology, Haimen, China) for $2 \mathrm{~h}$ at room temperature. The images from five fields of each ischemic region, from six rats in each group, were examined using the same brightness and exposure settings.

Western blot analysis. Twenty-four hours after retinal ischemia, fresh retinal tissues were homogenized on ice in cold lysis buffer (Beyotime Institute of Biotechnology) with a 
1:100 volume of phenylmethyl sulfonylfluoride (PMSF). The homogenates were centrifuged at $14,000 \mathrm{x}$ g for $5 \mathrm{~min}$ at $4^{\circ} \mathrm{C}$. The supernatants were aliquoted and stored at $-80^{\circ} \mathrm{C}$ following the removal of a small aliquot for protein estimation. Protein concentrations were determined using a bicinchoninic acid (BCA) protein assay kit (Beyotime Institute of Biotechnology). The samples were thawed on ice and mixed with $4 \mathrm{X}$ sample buffer (Invitrogen Life Technologies, Carlsbad, CA, USA), before heating at $100^{\circ} \mathrm{C}$ for $5 \mathrm{~min}$. Equivalent quantities of proteins $(50 \mu \mathrm{g})$ were loaded on $12 \%$ Tris-glycine sodium dodecyl sulfate (SDS)-polyacrylamide gels for fractionation at 160 V. Predetermined molecular weight standards (Beyotime Institute of Biotechnology) were used as markers. Protein on the gel was blotted onto nitrocellulose membranes at $106 \mathrm{~V}$ for $70 \mathrm{~min}$ at $4^{\circ} \mathrm{C}$. Following transfer, the membranes were incubated with blocking buffer $(5 \%$ skim milk in wash buffer) for $2 \mathrm{~h}$ at room temperature and washed three times (5 min/wash) with $0.1 \%$ Tris-buffered saline with Tween 20 (TBST). Incubation with occludin antibody in diluent buffer (5\% bovine serum albumin and $0.1 \%$ TBST) was performed overnight at $4^{\circ} \mathrm{C}(1: 1,000$ dilution). The membranes were then washed three times ( 5 min each) with TBST. The primary antibody was probed with horseradish peroxidase-conjugated IgG goat anti-rabbit secondary antibody $(1: 2,000)$ for $2 \mathrm{~h}$, washed three times in TBST and processed with enhanced chemiluminescence (ECL) detection reagents (Beyotime Institute of Biotechnology). The processed membranes were then exposed to photographic films for visualization of the signals. $\beta$-actin western blot analysis was performed for each membrane as an internal control of protein loading.

Evaluation of drug interactions. The interaction between escin and TA was analyzed using Berenbaum's method, to determine whether the combination was synergistic. The method is performed based on the following equation: $\mathrm{E}(\mathrm{da}, \mathrm{db})>\mathrm{E}(\mathrm{da})+\mathrm{E}(\mathrm{db})$, where $\mathrm{E}$ is the observed effect and $\mathrm{da}$ and $\mathrm{db}$ are the doses of agents a and $\mathrm{b}$, respectively. Synergism is indicated when the total effect of a combination is greater than expected from the sum of its effects (17).

Statistical analysis. Quantitative data from the experiments were expressed as the mean \pm standard deviation and significance was determined by one-way analysis of variance (ANOVA) followed by Tukey's test. In all cases, $\mathrm{P}<0.05$ was considered to indicate a statistically significant difference.

\section{Results}

Effects of escin and TA on BRB permeability. The IR rats demonstrated a greater level of retinal Evans blue leakage than the sham rats $(\mathrm{P}<0.01)$. High doses $(\mathrm{H})$ of escin $(1.8 \mathrm{mg} / \mathrm{kg})$ and TA $(5 \mu \mathrm{l})$ significantly inhibited retinal Evans blue leakage compared with the IR group $(\mathrm{P}<0.05$ and $\mathrm{P}<0.01$, respectively; Fig. 2). Low doses (L) of escin $(0.9 \mathrm{mg} / \mathrm{kg})$ and TA $(2 \mu \mathrm{l}) \mathrm{did}$ not significantly affect retinal Evans blue leakage compared with the IR group; however, administered together, they significantly inhibited retinal Evans blue leakage (Table I).

Effects of escin and TA on retinal thickness. The retinal thickness in the IR group was significantly increased compared
Table I. Effects of the combination of escin and TA on blood-retinal barrier permeability.

\begin{tabular}{lcc}
\hline Group & Evans blue (ng/mg) & Inhibition rate (\%) \\
\hline Sham & $14.1 \pm 10.2$ & - \\
IR & $47.0 \pm 17.2^{\mathrm{a}}$ & - \\
TA (L) & $34.6 \pm 8.3$ & 26.4 \\
Escin (L) & $37.9 \pm 16.3$ & 19.3 \\
TA (L) and escin (L) & $24.9 \pm 8.5^{\mathrm{b}}$ & $47.1^{\mathrm{c}}$ \\
\hline
\end{tabular}

${ }^{\mathrm{a}} \mathrm{P}<0.01$ vs. the sham group; ${ }^{\mathrm{b}} \mathrm{P}<0.05$ vs. the IR group; ${ }^{\mathrm{c}} \mathrm{E}(\mathrm{da}, \mathrm{db})>\mathrm{E}(\mathrm{da})+\mathrm{E}(\mathrm{db})$. IR, ischemia-reperfusion; TA, triamcinolone acetonide; L, low-dose. Data are expressed as the mean \pm standard deviation; $n=6$ per group.

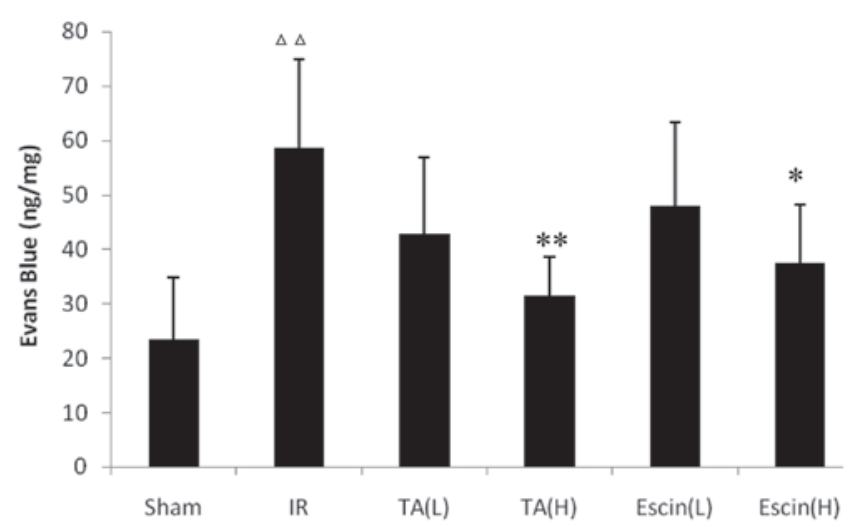

Figure 2. Effects of escin and triamcinolone acetonide (TA) on blood-retinal barrier (BRB) permeability. Escin and TA dose-dependently inhibited retinal Evans blue leakage. IR, ischemia-reperfusion; TA, triamcinolone acetonide; L, low-dose; H, high-dose. Data are expressed as the mean \pm standard deviation; $\mathrm{n}=6$ per group. ${ }^{*} \mathrm{P}<0.05$ and ${ }^{* *} \mathrm{P}<0.01$ vs. the IR group; ${ }^{\Delta \Delta} \mathrm{P}<0.01$ vs. the sham group.

with that of the sham group $(\mathrm{P}<0.05)$. Escin $(\mathrm{H})$ and TA $(\mathrm{H})$ significantly reduced retinal thickness compared with the IR group $(\mathrm{P}<0.05)$. There were no significant differences in retinal thickness between the TA $(\mathrm{L})$, escin $(\mathrm{L})$ and IR retinas (Fig. 3).

Effects of escin and TA on occludin expression following retinal ischemia. Escin (L) and TA (L) did not enhance occludin expression compared with IR alone. However, when escin and TA were administered together, occludin expression in the ganglion cell layer increased significantly, as demonstrated by the immunohistochemistry results (Fig. 4A and B). Western blot analysis produced consistent results (Fig. 4C).

\section{Discussion}

The retina is a highly specialized neural tissue that requires a unique vascular structure and tight control of permeability to allow correct visual function. Regulation of the flux of blood-borne metabolites into the retina is controlled by the BRB. Hypoxia, altered blood flow and retinal ischemia may 

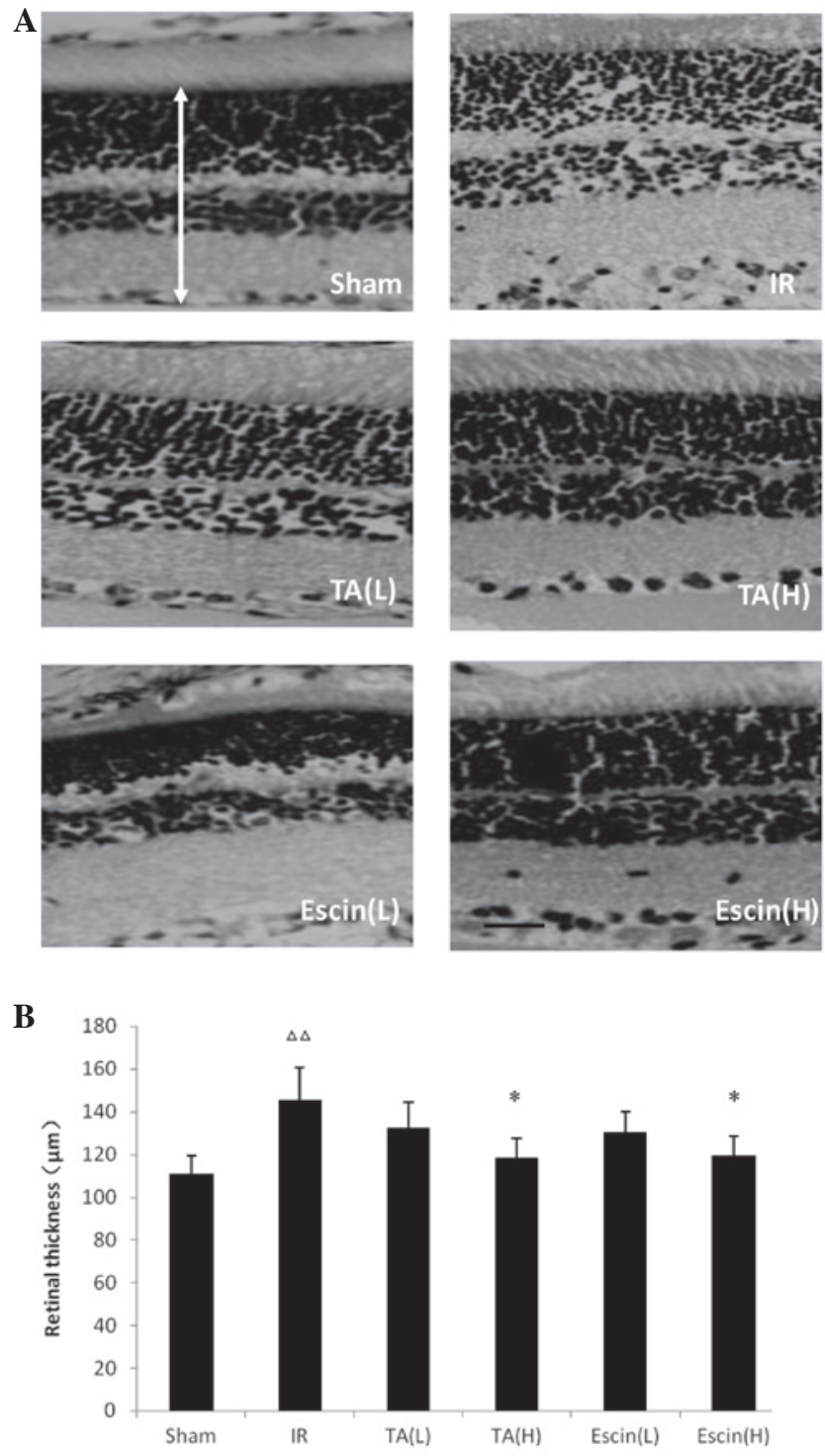

Figure 3. Retinal thickness was measured on hematoxylin and eosin (H\&E)-stained sections at the same location in each sample. (A) Micrograph showing representative retina samples from the different groups. The white arrow in the photograph of the control (sham) group demonstrates one measurement of retinal thickness. (B) Retinal thickness in the different groups. IR, ischemia-reperfusion; TA, triamcinolone acetonide; L, low-dose; $\mathrm{H}$, high-dose. Data are expressed as the mean \pm standard deviation; $\mathrm{n}=5$ per group. ${ }^{*} \mathrm{P}<0.05$ vs. the IR group and ${ }^{\Delta \Delta} \mathrm{P}<0.01$ vs. the sham group. Scale bar, $40 \mu \mathrm{m}$.

lead to BRB breakdown and the development of macular edema. Numerous experimental studies have demonstrated that ischemia is capable of inducing retinal damage (18). The retina is highly vulnerable to ischemic injury and its ischemic tolerance is $\leq 60 \mathrm{~min}$ (19).

In the present study, a retinal ischemia model was established in rats, in which ischemia was induced by increasing the IOP. The results revealed that BRB permeability and retinal thickness significantly increased following retinal ischemia. Treatment with escin (L) or TA (L) alone did not inhibit BRB permeability; however, when administered together, they significantly reduced BRB permeability following ischemia. This indicates that escin and TA have synergistic effects on reducing BRB permeability.
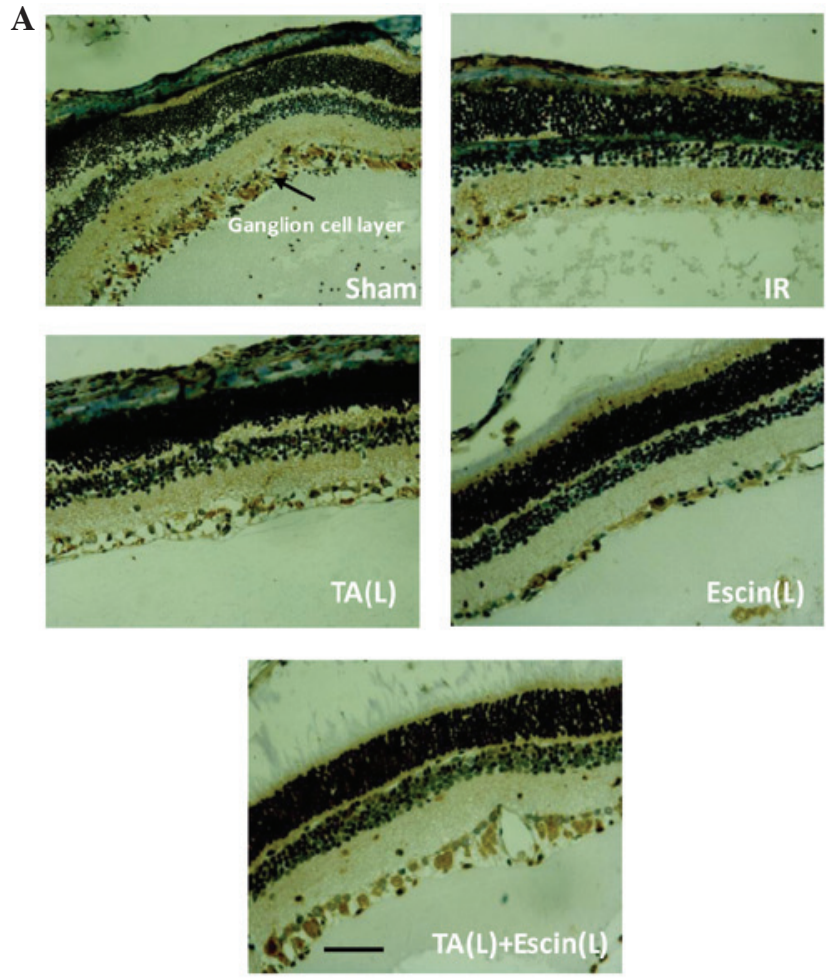

B

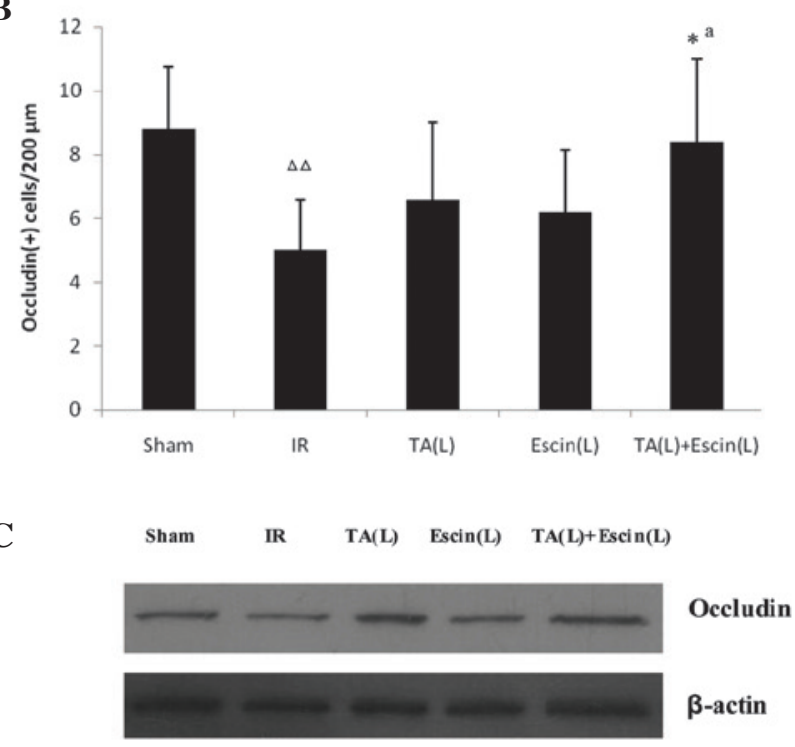

Figure 4. Effects of escin and triamcinolone acetonide (TA) on occludin expression in the retina following ischemia. Immunohistochemistry results: (A) Positive signals for occludin were localized in the retinal ganglion cell layer; and (B) low doses of escin and TA did not increase occludin expression compared with the IR group; however, when escin and TA were administered together, the occludin expression in the ganglion cell layer significantly increased. Western blot analysis results: (C) When escin and TA were administered together, the occludin expression increased significantly compared with when they were administered alone. Data are expressed as the mean \pm standard deviation; $n=3$ per group. ${ }^{*} \mathrm{P}<0.05$ vs. the IR group and ${ }^{\Delta \Delta} \mathrm{P}<0.01$ vs. the sham group. ${ }^{\mathrm{a}} \mathrm{E}(\mathrm{da}, \mathrm{db})>\mathrm{E}(\mathrm{da})+\mathrm{E}(\mathrm{db})$. Scale bar, $40 \mu \mathrm{m}$. IR, ischemia-reperfusion; TA, triamcinolone acetonide; L, low-dose; E, observed effect; da, dose of agent $a$; db, dose of agent $b$.

The BRB is composed of tight and adherent junction complexes. Retinal vascular endothelium and pigment epithelium have well-developed tight junctions that confer a high degree of control on solute and fluid permeability, thus 
maintaining the neural environment of the retina. Occludin is an important transmembrane protein in tight junctions that is responsible for forming the permeability barrier (20). In rats, the tight junction protein content is reduced following retinal ischemia (21). There is an inverse correlation between the tight junction protein content and endothelial permeability. Therefore, in vivo reduction of the tight junction protein content correlates with increased vascular permeability. The immunohistochemistry and western blot analysis results of the present study demonstrated that escin and glucocorticoids have synergistic protective effects on occludin.

In conclusion, escin and GCs were demonstrated to have synergistic protective effects on BRB breakdown; the molecular mechanism of which is correlated with the upregulation of occludin. Administration of escin may allow a reduction in the dose of GCs for the treatment of macular edema. The combination of escin with GCs is a potentially beneficial method of treatment for BRB breakdown and requires further study.

\section{References}

1. Williams R, Airey M, Baxter H, Forrester J, Kennedy-Martin $\mathrm{T}$ and Girach A: Epidemiology of diabetic retinopathy and macular oedema: a systematic review. Eye (Lond) 18: 963-983, 2004.

2. Ehrlich R, Harris A, Ciulla TA, Kheradiya N, Winston DM and Wirostko B: Diabetic macular oedema: physical, physiological and molecular factors contribute to this pathological process Acta Ophthalmol 88: 279-291, 2010.

3. Stewart MW: Corticosteroid use for diabetic macular edema: old fad or new trend? Curr Diab Rep 12: 364-375, 2012.

4. Liu L, Wu X, Geng J, Yuan Z and Chen L: IVTA as adjunctive treatment to PRP and MPC for PDR and macular edema: a meta-analysis. PLoS One 7: e44683, 2012.

5. Wilson CA, Berkowitz BA, Sato Y, Ando N, Handa JT and de Juan E Jr: Treatment with intravitreal steroid reduces blood-retinal barrier breakdown due to retinal photocoagulation. Arch Ophthalmol 110: 1155-1159, 1992.

6. Felinski EA, Cox AE, Phillips BE and Antonetti DA: Glucocorticoids induce transactivation of tight junction genes occludin and claudin-5 in retinal endothelial cells via a novel cis-element. Exp Eye Res 86: 867-878, 2008.
7. Jonas JB, Kreissig I and Degenring R: Intraocular pressure after intravitreal injection of triamcinolone acetonide. Br J Ophthalmol 87: 24-27, 2003.

8. Jonas JB, Degenring R, Vossmerbauemer U and Kamppeter B: Frequency of cataract surgery after intravitreal injection of high-dosage triamcinolone acetonide. Eur J Ophthalmol 15: 462-464, 2005 .

9. Fu F, Hou Y, Jiang W, Wang R and Liu K: Escin: inhibiting inflammation and promoting gastrointestinal transit to attenuate formation of postoperative adhesions. World J Surg 29: 1614-1620, 2005.

10. Zhang L, Fu F, Zhang X, Zhu M, Wang T and Fan H: Escin attenuates cognitive deficits and hippocampal injury after transient global cerebral ischemia in mice via regulating certain inflammatory genes. Neurochem Int 57: 119-127, 2010.

11. Wang T, Fu F, Zhang L, Han B, Zhu M and Zhang X: Effects of escin on acute inflammation and the immune system in mice. Pharmacol Rep 61: 697-704, 2009.

12. Zhang L, Wang H, Fan H, et al: The potent anti-inflammatory agent escin does not increase corticosterone secretion and immune cell apoptosis in mice. Fitoterapia 82: 861-867, 2011.

13. Xin W, Zhang L, Sun F, et al: Escin exerts synergistic anti-inflammatory effects with low doses of glucocorticoids in vivo and in vitro. Phytomedicine 18: 272-277, 2011.

14. Gong YY, Yu SQ, Wang H, et al: Clinical therapy of retinal vein occlusion with aescuven forte. Chin J New Drugs Clin Rem 24: 965-967, 2005 (In Chinese).

15. Wang WJ, Gong YY, Wang H, et al: Aescuven forte in treating central serous chorioretinopathy. Chin J New Drugs Clin Rem 24: 961-964, 2005 (In Chinese).

16. Yuan $\mathrm{Y}$ and $\mathrm{Wu} \mathrm{L}$ : Clinical observation of the central serous chorioretinopathy treated by aescinate sodium tablets combined with argon laser. Guiding Journal of TCM and Pharmacology 15: 58-59, 2009 (In Chinese).

17. Berenbaum MC: What is synergy? Pharmacol Rev 41: 93-141, 1989

18. Tsujikawa A, Ogura Y, Hiroshiba N, et al: Retinal ischemia-reperfusion injury attenuated by blocking of adhesion molecules of vascular endothelium. Invest Ophthalmol Vis Sci 40: 1183-1190, 1999.

19. Yilmaz T, Celebi S and Kükner AS: The protective effects of melatonin, vitamin $\mathrm{E}$ and octreotide on retinal edema during ischemia-reperfusion in the guinea pig retina. Eur $\mathrm{J}$ Ophthalmol 12: 443-449, 2002.

20. Furuse M, Hirase T, Itoh M, et al: Occludin: a novel integral membrane protein localizing at tight junctions. J Cell Biol 123: 1777-1788, 1993.

21. Xu HZ and Le YZ: Significance of outer blood-retina barrier breakdown in diabetes and ischemia. Invest Ophthalmol Vis Sci 52: 2160-2164, 2011. 